\title{
The myth of 'stable' coronary artery disease
}

Keith A. A. Fox ${ }^{1}$ Marco Metra, ${ }^{2}$ João Morais ${ }^{3}$ and Dan Atar ${ }^{4}$

\section{Affiliations}

${ }^{1}$ British Heart Foundation Centre for Cardiovascular Sciences, University of Edinburgh, Edinburgh, Scotland, United Kingdom. ${ }^{2}$ University of Brescia, Brescia, Italy. ${ }^{3}$ Leiria Hospital Centre, Leiria, Portugal. ${ }^{4}$ Oslo University Hospital, Dept. of Cardiology B, Oslo, Norway

\section{Correspondence to:}

Name: Keith Fox

Address: Centre for Cardiovascular Science, University of Edinburgh, 49 Little France Crescent, Edinburgh EH16 4S, UK

Phone no: 01312426378

Fax no: +441312426379

Email:k.a.a.fox@ed.ac.uk

Word count: 5759

Figures and tables: 2 Tables; 6 Figures

References: 114 


\section{Abstract}

Patients with known cardiovascular (CV) disease who have not suffered a recent acute event are often referred to as having 'stable coronary artery disease (CAD)'. The concept of 'stable' $C A D$ is misleading for two key reasons: the continuing risks of CV events over the longer term, and the diverse but powerful spectrum of risk characteristics. The risks of CV events are frequently underestimated and occur despite current standards of care for secondary prevention, including lifestyle changes, optimal medical therapy, myocardial revascularization, and use of antiplatelet agents to limit thrombosis. In dispelling the myth of 'stable' CAD, we explore the pathophysiology of the disease and the relative contribution of plaque and systemic factors to CV events. A broader concept of the vulnerable patient, not just the 'vulnerable' plaque, takes into account the diversity and future risks of atherothrombotic events. We also evaluate new and ongoing research into medical therapies aimed at further reducing the risks of CV events in patients with chronic but not 'stable' atherothrombotic disease.

Word count: 166

\section{Keywords}

Acute coronary syndrome; atherosclerosis; cardiovascular; coronary artery disease; myocardial infarction; peripheral artery disease

\section{Key points}

- The clinical label 'stable' coronary artery disease (CAD) needs to be reconsidered and more clearly defined, with chronic vascular disease including patient groups at substantial risk of future coronary events

- The risk of cardiovascular (CV) events in patients with chronic CAD is compounded by the presence of combined systemic and specific vascular risk factors

- Chronic CAD requires optimal medical therapy to mitigate the impact of modifiable risk factors and to reduce the risk of CV events (e.g. myocardial infarction, stroke, and CV death)

- Novel approaches may have the potential to further reduce the risk of adverse events, including profound lipid-lowering, inflammation-modifying agents, and novel antithrombotic combinations 
- Recent advances demonstrate that chronic vascular risk is modifiable and has the potential to produce clinically worthwhile gains in the most susceptible patients

- The field will continue to evolve, with improved characterization of patients at the highest risk of vascular events 


\section{Introduction}

Patients with known cardiovascular (CV) disease who have not suffered a recent acute event are often referred to as having 'stable coronary artery disease (CAD)'. However, how stable is 'stable CAD'? The term encompasses a diverse spectrum of patients. This includes patients with recurrent transient episodes of ischaemia induced by oxygen supply-demand imbalance in the presence of established coronary artery stenoses (i.e. stable angina and silent ischaemia), and those who have stabilized after an acute coronary syndrome (ACS), a phase that is often asymptomatic ${ }^{1}$. However, these apparently 'stable' patients are heterogeneous. CAD progression is dynamic and unpredictable and can unexpectedly lead to major adverse CV events (MACE; e.g. CV death, myocardial infarction [MI], and stroke) ${ }^{1}$. Of concern, patients remain at high risk of MACE despite adherence to current guidelinerecommended secondary prevention therapies ${ }^{2-6}$. The probability of having MACE within 5 years of the onset of apparently stable angina ranges up to $35 \%$ depending on clinical variables that affect the risk (FIG. 1) ${ }^{7}$. This diversity of risk highlights the need to identify those patients at higher risk and to further optimize their therapeutic management. The allembracing term 'stable' CAD masks those with chronic atherosclerotic disease and the amplified risk of adverse cardiovascular events.

This review aims to dispel the myth of 'stable' CAD by exploring our current understanding of CAD and to evolve the concepts from individual culprit or 'vulnerable' lesions toward the 'vulnerable patient' and the factors that identify such patients. This review will also identify indicators of advanced atherosclerotic disease, contributing to plaque instability, and risk factors that contribute to the systemic risk of thrombosis at sites of plaque erosion or rupture (e.g. diabetes, chronic kidney disease [CKD], etc.). Progress has been made in the development of risk stratification tools and there are implications for new therapies to optimize disease management. To avoid misconceptions around 'stable' CAD we apply the term 'chronic CAD' in place of 'stable CAD' in this review. By definition, the term 'chronic' characterizes an illness persisting for a long time or that is constantly recurring, i.e. the reality of long-term atherothrombotic disease.

\section{Pathophysiology}

The clinical manifestations of chronic CAD reflect the distribution and severity of vascular obstructive lesions. Both obstructive and non-obstructive lesions can lead to MACE and other significant CV events (e.g. unstable angina) ${ }^{1,8}$. Typically, such events are the result of 
atherothrombosis with disruption of an atheroma (i.e. rupture or erosion), provoking the formation of a thrombus that can interrupt blood flow locally or embolize (FIG. 2A) ${ }^{8}$. The probability of CV events depends upon several factors, including the extent and severity of atherosclerosis (being a diffuse/systemic disease), vulnerability of plaques to disruption, and the likelihood of thrombus formation and propagation (FIG. 2B). CV risk factors (such as diabetes, CKD, hypertension, and hyperlipidaemia) heighten the probability of an event. Hence, the clinical presentation and significance of atherothrombosis is variable ${ }^{1,9,10}$. Furthermore, because atherothrombosis is a systemic condition, a patient with CAD may also be at risk of stroke from cerebrovascular artery disease or an acute limb event from peripheral artery disease (PAD), or vice versa. In the REACH registry (an international, prospective, observational study enrolling patients with established CAD, cerebrovascular disease, or PAD, or with at least three atherosclerotic risk factors), $25 \%$ of patients had manifestations of thrombosis in more than one arterial bed ${ }^{11}$.

Autopsy studies suggested that $>70 \%$ of coronary thrombi develop superimposed on a ruptured atherosclerotic plaque ${ }^{8}$. In a minority of patients $(23 \%)$ who survived an $\mathrm{Ml}$, plaque erosion was identified by optical coherence tomography as the responsible precursor ${ }^{12}$. Atherothrombosis provoked by erosion of calcified nodules is rare ${ }^{13,14}$. Extensive research has focused on improving the ability to detect rupture-prone plaques. Thin-capped fibroatheromas (TCFAs) have been linked with clinically relevant thrombotic events; for example, Kubo $\mathrm{T}$ et al, demonstrated that $>80 \%$ of ruptured plaques that caused $\mathrm{MI}$ were TCFAs ${ }^{12}$. TCFAs have a large necrotic core covered by a thin fibrous cap and are accompanied by a loss of smooth muscle cells. Pro-inflammatory macrophages and other leukocytes may contribute to the degradation of the fibrous cap and its eventual rupture ${ }^{8}$. In the PROSPECT trial of patients with ACS who underwent three-vessel coronary angiography and intravascular ultrasound imaging after percutaneous coronary intervention (PCI), lesions associated with recurrent events were more likely to be characterized as TCFAs, compared with those not associated with recurrent events ${ }^{15}$. On the other hand, progress in secondary prevention measures, e.g. use of statins for lipid lowering, hypertension control, and smoking cessation, may have modified the characteristics of a typical atherosclerotic plaque, influencing the incidence of plaque rupture and increasing the incidence of erosion-induced thrombi. ${ }^{16}$ This may explain the recently observed increase in non-ST-elevation MI and decrease in ST-elevation MI in countries such as the US ${ }^{17,18}$, whereas ST-elevation MI still accounts for the majority of acute $\mathrm{Ml}$ events in many low- and middle-income countries ${ }^{19,20}$ albeit non-ST-elevation $\mathrm{MI}$ is on the increase in countries such as China ${ }^{20}$. 
Research has identified the contribution of non-culprit lesions and that of non-obstructive lesions (more prevalent than obstructive lesions). In the PROSPECT trial, MACE occurring during the 3-year follow-up was equally attributable to recurrence at the site of the culprit lesions versus non-culprit lesions (12.9\% and $11.6 \%$, respectively) ${ }^{15}$. Per lesion, obstructive lesions are more susceptible to rupture, but non-obstructive lesions are much more prevalent, and overall, they make a major contribution to the risk of ongoing vascular events $^{21}$. A key reason why targeting individual lesions with revascularization may fail to improve prognosis is due to the systemic nature of atherothrombosis ${ }^{22}$. Furthermore, not all plaque ruptures are symptomatic, so subclinical or 'non-culprit' ruptures are often undiagnosed, and their importance underestimated ${ }^{10}$. One pathological study observed that repeated plaque ruptures that heal are frequently found in men who die suddenly ${ }^{23}$. Plaque morphology is dynamic, with longitudinal imaging studies demonstrating that plaques can gain and lose characteristics of vulnerability over a period of months ${ }^{24}$. These findings support the need to focus on the 'vulnerable patient', not just the 'vulnerable lesion', and they highlight the need for effective systemic secondary prevention strategies beyond the current standard of care.

Continued advancement in imaging techniques and other methods to measure baseline disease burden will likely assist in improving risk prediction models for MACE. These include intravascular (intravascular ultrasound, optical coherence tomography, and plaque elastography) and non-invasive imaging. The latter includes advanced computerized tomography (CT) imaging and novel studies using ${ }^{18} \mathrm{~F}$-fluoride positron emission tomography combined with CT imaging ${ }^{25-27}$. Inflammatory markers and genetic risk factors (e.g. the 9p21 locus) are associated with atherothrombotic risk and susceptibility to recurrent plaque rupture $^{28}$. High-sensitivity cardiac troponin assays and newer biomarkers (including interleukins, growth factors, and thrombosis markers) can be used to predict the pre-test probability of obstructive $C A D^{29-32}$. The finding of elevated high-sensitivity troponin in patients with chronic CAD reflects the instability of this condition, given that troponin is a marker of myocyte necrosis, and illustrates the possible contribution of coronary micro-emboli to the progression of disease in 'chronic' vascular patients (although elevated troponin levels can also be a manifestation of left ventricular dysfunction and other atherosclerotic processes) ${ }^{33}$.

\section{Current guideline recommendations}

Guidelines from the European Society of Cardiology (ESC) and the American College of Cardiology/American Heart Association (ACC/AHA) provide a substantial set of measures to relieve symptoms of $C A D$ and improve prognosis through prevention of $C V$ events ${ }^{1,34}$. In line 
with our current understanding of the pathophysiology of atherothrombosis, recommendations for the prevention of MACE encompass: 1) the control of CV risk factors to limit progression of atherosclerosis and stabilize existing plaques; and 2) the prevention of thrombus formation over ruptured or eroded plaques (FIG. 3).

The control of $\mathrm{CV}$ risk factors includes both lifestyle modifications and medical interventions, supported by patient education. Guidelines recommend smoking cessation, regular physical exercise, adopting a healthy diet, weight management, and psychosocial support ${ }^{1,34-39}$. Medical interventions for CV risk factors encompass lipid control, with statins recommended for all patients with CAD (other lipid-lowering therapies such as ezetimibe and approved PCSK9 monoclonal antibodies can be considered in select patients with intolerance or inadequate response to statins), and the use of an angiotensin-converting enzyme (ACE) inhibitor (or an angiotensin receptor blocker [ARB] as an alternative) to lower blood pressure in patients with CAD and hypertension, and therapies to improve CV outcomes in diabetes, and heart failure $(\mathrm{HF})^{1,34-37}$. In cases that are unresponsive to medical therapy, the 2014 ESC/European Association for Cardio-Thoracic Surgery (EACTS) guidelines on myocardial revascularization state that revascularization can be used to improve symptoms in patients with any coronary stenosis $>50 \%$ and is indicated for improved prognosis in patients with substantial stenosis/stenoses ( $>50 \%$ ) that are anatomically or functionally significant, particularly if in the left main artery or in cases of 2- or 3-vessel disease ${ }^{40}$. Guidance from the AHA/ American College of Cardiology Foundation (ACCF) states that revascularization is indicated to improve prognosis in survivors of out-of-hospital cardiac arrest and ventricular tachycardia and in patients with left ventricular dysfunction ${ }^{38}$. In a recent review by Katritsis et al, it was noted that "the evidence base in revascularization for stable CAD is fragmentary", and that "treatment recommendation should be formulated by a multidisciplinary approach from interventionalists, cardiac surgeons, and non-invasive cardiologists and from patients themselves". Clinical judgement is especially important for patients who are not typical representatives of any clinical trial ${ }^{41}$.

Antithrombotic agents are used in the prevention of thrombus formation, but the intensity of their use differs according to the clinical setting (MI or ACS, stent insertion, or chronic CAD). In current guidelines, antiplatelet agents are recommended to prevent the formation of coronary thrombus, specifically low-dose acetylsalicylic acid (ASA; aspirin) for medically managed patients with chronic $C A D$, with the $P 2 Y_{12}$ inhibitor clopidogrel recommended when ASA is not tolerated ${ }^{1,34}$. Dual antiplatelet therapy (DAPT) with a P2Y 12 inhibitor plus ASA is recommended in patients with chronic CAD for up to 1 year after coronary stent implantation, with ASA monotherapy used thereafter ${ }^{42}$. Data supporting a role for dual targeting of the 
platelet and coagulation pathways of thrombosis in patients with chronic CAD have been published recently ${ }^{4,43}$; however, current guidelines precede the latest research in this area.

\section{The persistent risk of events}

Patients are still at high residual risk of MACE despite guideline-recommended therapy. This is clearly demonstrated by the incidence of MACE in the reference arms of randomized controlled trials (RCTs) attempting to optimize the therapeutic management of chronic CAD with intensified secondary prevention strategies. Examples include RCTs evaluating intensified antithrombotic strategies versus standard of care antiplatelet therapy (PEGASUSTIMI 542, TRA $2^{\circ} \mathrm{P}-\mathrm{TIMI}^{5} 0^{3}$, and $\left.\mathrm{COMPASS}^{4}\right)$. In addition, the CANTOS trial, which evaluated the anti-inflammatory therapy canakinumab in patients with a history of $\mathrm{MI}$ and increased blood levels of high-sensitivity C-reactive protein (hsCRP), demonstrated a $4.5 \%$ annual risk of MACE in the placebo group receiving standard secondary prevention ${ }^{5}$. In the IMPROVE-IT trial, which evaluated the non-statin drug ezetimibe in patients following recent hospitalization for ACS, the 7-year risk of CV death, major coronary event (MI, unstable angina requiring hospitalization, or coronary revascularization) or non-fatal stroke was 35\% 6 . Across these trials, baseline use of secondary prevention strategies was high, with $\geq 72 \%$ of patients receiving an $A C E$ inhibitor/ARB, $\geq 92 \%$ a lipid-lowering therapy and $\geq 95 \%$ an antithrombotic therapy ${ }^{2-6}$.

The persistent risk of CV events in patients with chronic CAD is also shown by registries and other large-scale observational data. In the REACH registry, consistent with the RCT data above, the 1-year incidence of MACE was $4.5 \%$ in the overall CAD population enrolled in the REACH registry (Table 1$)^{44}$. In a retrospective cohort study of data from Swedish national registries, Jernberg et al. demonstrated that the risk of MACE persisted long after an index $\mathrm{Ml}$ regardless of an absence of events for the first 12 months; the cumulative probability of subsequent MACE (after 12 months of surviving without recurrent MI or stroke; $n=76,687$ ) was $9 \%$ at 12 months and $20 \%$ at 36 months (FIG. 4$)^{45}$.

These data highlight the need to improve what we refer to as 'optimal medical therapy' for the secondary prevention of MACE in patients with chronic CAD. Patients with chronic CAD who are well-managed with current secondary prevention therapies (based on current guidelines) have a persistent risk of MACE, especially among those with multiple CV risk factors. 


\section{The heterogenous risk of events}

As discussed in the 'Pathophysiology' section, two areas underpin a patient's risk of CV events: 1) the advanced/systemic nature of CAD, and 2) the presence of CV risk factors that impact on the progression and stability of atherosclerotic plaques and the risk of atherothrombosis (FIG. 2B). Advanced disease can be identified through the presence of stress-induced ischaemia (inferring obstructive disease), CT imaging, disease in multiple vascular beds, and prior revascularization as a marker of defined $C A D^{46}$. Risk factors, including patient characteristics such as age and sex, and comorbidities including diabetes, $\mathrm{CKD}, \mathrm{HF}$, dyslipidaemia, and hypertension, can amplify the baseline CV risk, and the risk of atherosclerotic disease the risk of MACE. In turn, these factors increase the potential for thrombotic CV complications (Supple FIG. 1) ${ }^{7,10,47}$. The prevalence of important comorbidities has been demonstrated to be high among patients with chronic CAD (Table 2) (8) $^{\text {; a large }}$ database analysis demonstrated that comorbid disease is strongly associated with survival in patients with $\mathrm{CAD}^{49}$.

\section{Markers of advanced disease}

\section{'Stable’ angina}

Symptomatically 'stable' angina is defined as transient chest pain or tightness in the chest provoked by exertion or emotional stress that can be relieved by rest or administration of nitroglycerin ${ }^{1}$. Such symptoms are frequently the result of obstructive CAD, in which subclinical cycles of plaque disruption, thrombi formation, and subsequent healing can result in an accumulation of material over time and progressive lumen obstruction ${ }^{8}$. In the previously described $\mathrm{REACH}$ registry, $52 \%$ of patients with baseline documented chronic CAD had 'stable' angina, and the 4-year incidence of MACE in these patients was $16.3 \%$ compared with the $14.2 \%$ in patients without anginal symptoms. Furthermore, the difference in the risk of $\mathrm{Ml}$ and stroke remained significant with adjustment for baseline characteristics, including use of ASA and statins ${ }^{50}$.

\section{History of cardiovascular events}

The risk of recurrent events or death remains high in the first year following an event such as $\mathrm{Ml}$ or stroke. In a retrospective analysis of data collected from the Singapore National Registry of Disease Office, the adjusted odds of recurrence of the same event within a year was $6.8 \%$ in patients with $\mathrm{Ml}$ and $4.8 \%$ in patients with ischaemic stroke with the highest probability in the first 30 days following the index event. Mortality over the 1-year period was $31.7 \%$ and $17.1 \%$ in patients with $\mathrm{MI}$ and those with ischaemic stroke, respectively ${ }^{51}$. In an analysis of data from GRACE (a large, multinational registry enrolling patient with ACS), one- 
third of MACE occurred in the first 4 days following the qualifying ACS event; however, $66 \%$ of deaths and $59 \%$ of reinfarctions took place subsequently in the 6-month follow-up period ${ }^{52}$. In the CAPRIE study, the risk of MACE was increased in the subgroup of patients with prior events compared with the overall population (23.8\% and $15.2 \%$ at 3 years, respectively, in patients who received ASA ${ }^{53}$. Such evidence supports the guideline-recommended use of DAPT for up to 1 year after an ACS event ${ }^{42}$.

There is strong evidence, however, to support a persistent risk of MACE beyond 1 year after a CV event, both from observational studies ${ }^{45,48,54}$ and RCTs ${ }^{55-57}$ (FIG. 4). Such data underpin guideline recommendations to consider the use of DAPT beyond 1 year after an ACS event in patients who have tolerated DAPT without bleeding complications ${ }^{42}$.

\section{Disease in several vascular beds}

As discussed in the 'Pathophysiology' section atherosclerosis is a progressive, systemic disease. Therefore, if one vascular bed is found to be affected, it is likely that the others are too. As such, disease in several vascular beds is common; for example, $24.8 \%$ of patients with $C A D$ in the REACH registry had concomitant disease in other vascular beds ${ }^{11}$. This is associated with an increased risk of MACE and other significant CV events, as demonstrated in REACH ${ }^{44,58}$ and the randomized PEGASUS-TIMI 54 trial, in which the residual risk of MACE with ASA was highest in patients with CV disease in more than one vascular bed versus the overall population (9.4\% and $8.6 \%$, respectively $)^{59}$. As such, disease in more than one vascular bed has substantial prognostic power; for example, disease in both two and three vascular beds were found to be significant predictors of CV death and recurrent CV events at 20 months in a multivariable analysis used in the development of a risk stratification model based on REACH registry data (Table 2$)^{60}$.

\section{Revascularization}

Among patients with suspected chronic stable vascular disease, prior revascularization identifies those with defined vascular disease and is, therefore, a marker of disease severity. Crucially, these patients are still in need of medical secondary prevention strategies to reduce the risk of MACE. Several datasets provide examples of the high risk of MACE in revascularized versus non-revascularized patients (e.g. the CAPRIE ${ }^{61,62}$ and TRA $2^{\circ} \mathrm{P}-\mathrm{TIMI}$ $50^{63}$ trials). Furthermore, a large database analysis demonstrated that the risk of operative mortality was doubled in patients who required a second coronary artery bypass grafting (CABG) procedure compared with those who required a first-time CABG procedure. In the long term (up to 6 years postoperatively), overall survival was significantly lower in patients undergoing a second $\mathrm{CABG}$ versus a first $\mathrm{CABG}^{64}$. 


\section{Comorbidities}

\section{Type 2 diabetes mellitus}

Type 2 diabetes mellitus (T2DM) is a common comorbidity in patients with atherosclerotic disease and was shown to be present in $38 \%$ of patients (with 4-years' follow-up data) in the REACH registry ${ }^{65}$. T2DM is a major risk factor for MACE because it is associated with a greater thrombotic predisposition; atherosclerosis is more widely and diffusely distributed in the coronary arteries of patients with T2DM, and atheromas have a greater inflammatory infiltrate and a larger necrotic core size ${ }^{66}$. In the REACH registry, T2DM was associated with a $27 \%$ increase in the relative risk of MACE $(16.5 \%$ and $13.1 \%$ in patients with and without diabetes at 4 years, respectively $)^{65}$. Similar trends were reported from analysis of data from the reference arms of the CAPRIE and TRA $2^{\circ} \mathrm{P}-\mathrm{TIMI} 50 \mathrm{RCTs}$ (evaluating intensified antiplatelet regimens) ${ }^{67,68}$. Of note, some evidence suggests that patients with extensive CAD and T2DM might benefit from prompt over-delayed revascularization. The BARI 2D trial demonstrated that among 381 patients with T2DM and CAD with high-risk angiographic characteristics intended for CAGB, the 5-year risk of death, MI and stroke was significantly reduced in patients randomized to receive rapid coronary revascularization relative to those assigned to deferred revascularization in a setting of coordinated medical therapy $(24.8 \%$ and $36.8 \%$, respectively; $p=0.005)^{69}$.

\section{Chronic kidney disease}

CKD is more prevalent in patients with chronic CAD compared with the general population. One-third of outpatients at risk of atherothrombotic events in the $\mathrm{REACH}$ registry had moderate-to-severe CKD, and just under one-quarter of patients in CLARIFY had CKD ${ }^{70,71}$. This is similar to an estimated global prevalence between $11 \%$ and $13 \%$ in the general medical population globally, with the majority of patients in stage $3 \mathrm{CKD}^{72}$. This is because CKD is associated with accelerated CVD and a higher risk of MACE, mediated by factors including impaired clearance of pro-atherogenic cytokines and uremia-specific metabolites, and an increase in vascular calcification ${ }^{73,74}$. A patient with CKD is more likely to die from a CV-related cause than progress to end-stage kidney disease, with CVD accounting for more than $50 \%$ of mortality in patients with $\mathrm{CKD}^{74}$.

Analyses of data from the REACH and FRENA registries demonstrated that CKD severity was an independent predictor of the extent and severity of vascular disease ${ }^{70,75}$. In the PEGASUS-TIMI 54 trial, the risk of MACE was $14.0 \%$ in patients with an estimated 
glomerular filtration rate of $<60 \mathrm{~mL} / \mathrm{min} / 1.73 \mathrm{~m}^{2}$ compared with $9.0 \%$ in the overall population $^{76}$.

\section{Heart failure}

$\mathrm{HF}$ is the fastest-growing CV disease in terms of prevalence, affecting more than 60 million people worldwide, and it is associated with a substantial morbidity, mortality, and economic burden $^{77,78}$. CAD is a major contributor to the development of HF; it is estimated that at least two-thirds of patients with HF have underlying $C A D^{79}$. HF is also associated with an increased risk of MACE in patients with concomitant CAD. For example, in the REACH registry, congestive $\mathrm{HF}$ was associated with a $\sim 70 \%$ increase in the risk of $\mathrm{MACE}^{54}$.

\section{Risk stratification}

Comorbidities frequently occur together in patients with CAD (with many being independent risk factors for each other). An accumulation of $C V$ risk factors elevates the risk of MACE and other manifestations of CAD such as HF. For example, patients with T2DM and HF have a particularly high risk of $\mathrm{CV}$ death ${ }^{65}$; patients with T2DM, congestive HF, and disease in more than one vascular bed have an elevated risk of MACE ${ }^{65}$; the coexistence of T2DM and hypertension significantly increases the risk of $\mathrm{HF}^{80}$; and following $\mathrm{PCl}$, patients with T2DM have worse outcomes than those without T2DM ${ }^{81,82}$.

Current guidelines recommend a pyramidal structure to prognostic assessment, to categorize patients into a high, intermediate, or low risk of all-cause death. In-sequence risk assessment is carried out by clinical evaluation, ventricular function, response to stress testing, and coronary anatomy'. As discussed previously in the 'Pathophysiology' section, only select high-risk patients are likely to benefit in terms of survival from revascularization. After revascularization, patients need to be managed with global CV risk control strategies and antithrombotic event prevention ${ }^{40}$.

Several scores may be used to predict the risk of MACE in patients with established or suspected chronic CAD, but their use is limited in clinical practice (Table 1).

The concept of 'personalized' or 'precision' medicine has the potential to improve risk stratification and improve the application of secondary prevention therapies. These approaches may incorporate improved imaging, biomarkers, and genetic markers in conjunction with optimized therapeutic strategies ${ }^{32}$. However, the clinical impact of the use of 
such methods is still unsettled and the era of precision medicine has not yet emerged for chronic CAD.

\section{Optimizing the therapeutic management}

Standard secondary prevention strategies are evolving to address the high residual risk of MACE observed in patients with chronic CAD despite their use. These advancements include the development of new antithrombotics, lipid-lowering drugs, and anti-inflammatory agents.

\section{Antithrombotic therapy}

Platelets have long been recognized as central to the development of arterial thrombosis ${ }^{83,84}$. Several RCTs have attempted to maximize reductions in the risk of MACE via intensified


a prior event ${ }^{2,53,86}$, disease in more than one vascular bed ${ }^{59,87}, \mathrm{~T}^{2} \mathrm{DM}{ }^{67,68,88}$, and CKD ${ }^{89}$. Despite the incremental improvements in the prevention of vascular events with intensifying antiplatelet therapy, a substantial residual risk of MACE remained despite high use of additional secondary prevention medications ( $\geq 91 \%$ statins/lipid-lowering therapy; $74-90 \%$ ACE inhibitors/ARBs) ${ }^{2,3,85}$. Furthermore, there was an increased risk of major bleeding events and/or a lack of mortality benefit across these trials ${ }^{2,3,61,85}$ (FIG. 5).

Attempts to further optimize antithrombotic strategies for chronic-phase CV disease are increasingly focused upon dual targeting of both the antiplatelet and coagulation pathways. Coagulation is now understood to play a key role in arterial clot stabilization and the amplification of the platelet activation by thrombin and the role of platelet thrombin receptors in coagulation are increasingly recognized. Hence, it is possible that synergies exist between platelet inhibition and anticoagulation ${ }^{83}$. The first trials to evaluate anticoagulants outside of the acute setting were focused on vitamin K antagonists (VKAs). Meta-analyses of such studies demonstrated a reduction in the risk of CV events with VKA therapy compared with ASA alone (e.g. the annual risk of recurrent MI was reduced by $44 \%)^{90}$ and suggested improved efficacy benefits with VKA use compared with clopidogrel plus ASA therapy ${ }^{91}$. However, in these meta-analyses, the benefits were offset by a more than twofold increase in the incidence of major bleeding events ${ }^{90,91}$. The development of non-VKA oral anticoagulants (NOACs) led to trials of NOACs in patients with ACS (APPRAISE-2, ATLAS ACS 2 TIMI 51, and GEMINI ACS 1) ${ }^{92-94}$ and with chronic CAD/PAD (COMPASS) ${ }^{43}$. 
The phase III APPRAISE-2 trial evaluated the addition of the Factor Xa inhibitor apixaban to standard antiplatelet therapy or the prevention of MACE in patients after acute ACS at high risk of a recurrent ischaemic event. However, the study was stopped early because apixaban, at a dose of $5 \mathrm{mg}$ twice daily (bid; the full stroke prevention dose for patients with $\mathrm{AF}$ ), was associated with a significant increase in the risk of major bleeding compared with placebo, without a significant reduction on the incidence of recurrent ischaemic events ${ }^{92}$. The excess bleeding risk with apixaban may have been attributable to the dose selection the inclusion of patients with a high risk of bleeding in the trial ${ }^{95}$. The phase III, double-blind ATLAS ACS 2 TIMI 51 trial evaluated two doses of rivaroxaban, $2.5 \mathrm{mg}$ bid and $5 \mathrm{mg}$ bid, in patients with a recent ACS (unlike in APPRAISE-2, these doses were much lower than those indicated for patients with $\mathrm{AF})^{93}$. Both doses of rivaroxaban (with a background of ASA, or ASA plus clopidogrel or ticlopidine) significantly reduced the risk of MACE versus placebo at the expense of an increased risk of bleeding events (but not fatal bleeding events) versus placebo. The rivaroxaban $2.5 \mathrm{mg}$ bid dose had improved safety outcomes over the rivaroxaban $5 \mathrm{mg}$ bid dose ${ }^{93}$. These results led to European (but not North American) approval of the use of rivaroxaban $2.5 \mathrm{mg}$ bid for the secondary prevention of CV events after an ACS in patients with elevated biomarkers (co-administered with ASA alone or in combination with DAPT [ASA plus clopidogrel or ticlopidine] $)^{96,97 .}$

As a result of the findings from ATLAS ACS 2 TIMI 51, it was hypothesized that removal of ASA, to avoid a triple therapy regimen of DAPT plus an anticoagulant, might reduce the risk of bleeding relative to DAPT alone (as seen in the WOEST trial in a post-PCl setting with VKA plus clopidogrel versus triple therapy ${ }^{94,98}$. To investigate this hypothesis further, the double-blind, phase II GEMINI ACS 1 trial was conducted to evaluate the safety of rivaroxaban $2.5 \mathrm{mg}$ bid compared with low-dose ASA with a background of $\mathrm{P}_{2} \mathrm{Y}_{12}$ inhibitor therapy (clopidogrel or ticagrelor, at the investigators discretion) in patients with a recent ACS event ${ }^{94,98}$. Indeed, the risk of TIMI non-CABG clinically relevant bleeding was similar between treatment arms ( $5 \%$ with rivaroxaban versus $5 \%$ with ASA). Furthermore, the absence of ASA in the experimental arm had no observed impact on stent thrombosis (87\% of patients had $\mathrm{PCl}$ for the index event). Conversely, a substantially higher risk of stent thrombosis has been observed when $\mathrm{P}_{2} \mathrm{Y}_{12}$ inhibitors were stopped early after $\mathrm{PCl}$ for $\mathrm{ACS}^{94}$. A larger, more adequately powered trial would be needed to substantiate the findings from GEMINI ACS 1, and a better understanding of the intensity of antithrombotic therapy suitable for the transition from the acute to chronic setting is needed ${ }^{94}$. Of note, there are several ongoing studies evaluating antithrombotic regimens that exclude ASA in the setting of ACS (NCT03234114) and chronic CAD or PAD (NCT02567461; NCT02548650). 
In the chronic vascular disease setting, rivaroxaban has been recently investigated in patients with chronic CAD or PAD in the phase III COMPASS trial ${ }^{4,43,99}$. The results of this newest study provided further support of the potential benefit of a low dose of the Factor Xa inhibitor rivaroxaban in combination with antiplatelet therapy in patients with atherothrombosis. In total, 27,395 eligible patients with chronic CAD or PAD were randomized to receive rivaroxaban $2.5 \mathrm{mg}$ bid plus ASA $100 \mathrm{mg}$ once daily ( $n=9152$ ), rivaroxaban $5 \mathrm{mg}$ bid ( $\mathrm{n}=9117)$, or ASA $100 \mathrm{mg}$ once daily ( $\mathrm{n}=9126)$ in addition to guidelinerecommended secondary preventative therapies. In patients receiving rivaroxaban $2.5 \mathrm{mg}$ bid plus ASA, the risk of MACE was significantly reduced by $24 \%$ compared with those receiving $A S A$ alone ( $4.1 \%$ and $5.4 \%$, respectively; $H R=0.76 ; p<0.001$ ), supporting dual targeting of the platelet and coagulation pathways of thrombosis. Overall, the risk of major bleeding was increased by $70 \%$ in patients receiving rivaroxaban $2.5 \mathrm{mg}$ bid plus ASA therapy versus those receiving ASA alone (3.1\% and $1.9 \%$, respectively; $\mathrm{HR}=1.70 ; p<0.001)$. Rates of fatal bleeding ( $0.2 \%$ and $0.1 \%$, respectively) and $\mathrm{ICH}(0.3 \%$ and $0.3 \%$, respectively) were low and similar between the treatment arms. Unlike the studies of intensified antiplatelet therapy mentioned previously (i.e. CHARISMA, PEGASUS-TIMI 54, and TRA $2^{\circ} \mathrm{P}$-TIMI 50$)^{2,3,86}$, the COMPASS trial demonstrated a survival benefit with the addition of an anticoagulant ${ }^{43}$. In a pre-specified CAD subanalysis of the COMPASS data, the net clinical benefit outcome (defined as the composite of CV death, stroke, MI, fatal bleeding, or symptomatic bleeding into a critical organ) occurred in $4.7 \%$ of patients receiving rivaroxaban $2.5 \mathrm{mg}$ bid plus ASA versus $6.0 \%$ in those receiving ASA alone ( $\mathrm{HR}=0.78 ; p=0.0003)$. Results were consistent across a variety of patient subgroups, including patients with a history of MI, T2DM, and concurrent PAD ${ }^{4}$. In a subanalysis of data from 7470 patients with PAD or carotid artery disease (with or without CAD) enrolled in COMPASS, rivaroxaban 2.5 mg bid plus ASA reduced the risk of MACE and major adverse limb events including major amputation both as separate endpoints and as a combined composite outcome as follows: $6.3 \%$ and $2.4 \%$, respectively; $\mathrm{HR}=0.54 ; p=0.0037^{99}$. The external validity of results from the COMPASS trial has been evaluated using data from the REACH registry. According to this analysis, a substantial number of evaluable patients enrolled in REACH (52.9\%) would have been eligible for the COMPASS trial, demonstrating a sufficient level of external validity. COMPASS-eligible patients in REACH were at a significantly higher annualized risk of MACE than those enrolled in COMPASS $(4.2 \% \text { and } 2.9 \% \text {, respectively; } p<0.001)^{100}$.

\section{Lipid-lowering agents}

Statins are recommended for all patients with established CAD, with a treatment goal of LDL$\mathrm{C}<1.8 \mathrm{mmol} / \mathrm{L}$ ( $<70 \mathrm{mg} / \mathrm{dL}$ ) or $>50 \%$ LDL-C reduction when the target level cannot be 
reached. If the treatment goal is not reached, replacing or combining statins with other lipidlowering therapies becomes an option ${ }^{37}$. One such therapy is ezetimibe, which inhibits cholesterol absorption in the gut. In the IMPROVE-IT trial, the addition of ezetimibe to simvastatin versus simvastatin alone significantly lowered average LDL-C levels $(53.7 \mathrm{mg} / \mathrm{dL}$ and $69.5 \mathrm{mg} / \mathrm{dL}$, respectively; $p<0.001$ ) and the composite risk of MACE and unstable angina requiring hospitalization and revascularization (Kaplan-Meier event rate at 7 years: $32.7 \%$ and $34.7 \%$, respectively; $p=0.016)^{6}$.

Proprotein convertase subtilisin/kexin type 9 (PCSK9) is a serine protease involved in cholesterol homeostasis. PCSK9 binds to the LDL receptor complex inducing intracellular degradation, reducing serum LDL clearance. The subcutaneously injected PCSK9 monoclonal antibodies alirocumab and evolocumab are approved by the US Food and Drug Administration (FDA) and the European Medicines Agency (EMA) for the treatment of hypercholesterolemia in patients with intolerance or inadequate response to statins, especially in patients at very high risk of CV events or in the case of familial hypercholesterolemia ${ }^{37}$. A recent meta-analysis of the long-term efficacy and safety of PCSK9 antibodies demonstrated significantly decreased LDL-C levels (mean difference: $50.23 \%$; $95 \%$ confidence interval [Cl] $-56.65 \%$ to $-43.82 \%$ ) compared with no PCSK9 antibody treatment, and significant reductions in the rates of $\mathrm{Ml}$ (relative risk $[R R]=0.73 ; 95 \%$ $\mathrm{Cl} 0.65-0.82)$, coronary revascularization ( $\mathrm{RR}=0.79 ; 95 \% \mathrm{Cl} 0.73-0.87)$, and stroke (RR=0.81; 95\% Cl 0.68-0.96). There were no significant differences between the risk of treatment-emergent adverse events or serious treatment-emergent adverse events ${ }^{101}$.

It has been demonstrated that the level of LDL-C correlated with a decreased risk of events, without a clear threshold ${ }^{102}$. Studies also demonstrated that plaque regression by $1 \%$ over 12 months can be achieved with LDL-C within the range of $36-70 \mathrm{mg} / \mathrm{dL}^{103}$.

\section{Anti-inflammatory agents}

Research has suggested that inflammatory activity contributes to the vulnerability of plaque and that systemic inflammation may increase the risk of thrombosis at sites of plaque rupture or erosion ${ }^{9}$. Furthermore, biomarkers of inflammation, such as hsCRP and interleukin (IL)-6, are associated with an increased risk of CV events, independent of the cholesterol level. Statins have been shown to reduce C-reactive protein levels, and their anti-inflammatory effect has been demonstrated to contribute to the reduction of MACE in at-risk patients ${ }^{5}$. 
Canakinumab, an IL-1 $1 \beta$ inhibitor with proven anti-inflammatory effects, was assessed in the phase III CANTOS trial to evaluate the impact of lowering levels of hsCRP on the risk of MACE in patients with a history of $\mathrm{MI}$ and a persistent inflammatory response ${ }^{5}$. Canakinumab significantly reduced hsCRP levels from baseline, as compared with placebo, without reducing LDL-C levels, and the canakinumab $150 \mathrm{mg}$ dose resulted in a significantly lower incidence of recurrent CV events compared with placebo (3.9\% per year and $4.5 \%$ per year, respectively; $\mathrm{HR}=0.85 ; 95 \% \mathrm{Cl} 0.74-0.98 ; p=0.021)$. Canakinumab was associated with a higher risk of infection versus placebo but not all-cause mortality ${ }^{5}$. In contrast, in the CIRT trial of 4786 patients post $\mathrm{MI}$ or with multivessel CAD with either diabetes or metabolic syndrome, low-dose methotrexate (which targets a different component of the inflammatory pathway to canakinumab) did not reduce the levels of critical IL-1 $\beta$ to IL- 6 to CRP pathway of innate immunity nor lower the risk of MACE as compared with placebo ${ }^{104}$. Nonetheless, further targeting of IL-1 $\beta$ and other inflammatory pathways might further improve the residual risk of MACE observed with current secondary prevention strategies.

\section{Antidiabetic agents}

Clinical trials of antidiabetic agents demonstrate that improvement in glycemic control does not automatically reduce CV risk; in fact, an increase in CV risk has been observed with some agents, and as a result the FDA has mandated that the risk of MACE be evaluated with new diabetic drugs ${ }^{105}$. Since then, several agents have been demonstrated to significantly lower the risk of MACE versus placebo in randomized controlled trials: the sodium-glucose co-transporter 2 antagonist empagliflozin in the EMPA-REG OUTCOME trial (10.5\% vs $12.1 \%$, respectively; $H R=0.86 ; p=0.04)^{35}$, the glucagon-like peptide-1 (GLP-1) receptor agonist liraglutide in the LEADER trial $(13.0 \% \text { vs } 14.9 \% \text {, respectively; } H R=0.87 ; p=0.01)^{106}$, and the long-acting GLP-1 receptor agonist semaglutide in the SUSTAIN-6 trial $(6.6 \%$ vs $8.9 \%$, respectively; $\mathrm{HR}=0.74 ; p<0.001)^{107}$.

\section{Conclusions}

The clinical label 'stable' CAD needs to be reconsidered and more clearly defined as chronic coronary vascular disease, encompassing a diversity of patient groups including those at substantial risk of future coronary events. Chronic CAD requires optimal medical therapy to mitigate the impact of modifiable risk factors and to reduce the risk of CV events (e.g. MI, stroke, and CV death). Secondary prevention should include current guideline-indicated measures, such as lifestyle changes. Novel secondary prevention measures, which include profound lipid-lowering, inflammation-modifying agents and novel antithrombotic combinations, might have the potential to further reduce the risk of adverse events. The field 
will continue to evolve with improved characterization of patients at the highest risk of vascular events, and could include novel imaging techniques to differentiate those with the highest risk plaque subtypes and use of novel systemic biomarkers of vascular disease.

Recent advances demonstrate that chronic vascular risk is modifiable and that novel approaches in managing these risk factors has the potential to produce clinically worthwhile gains in the most susceptible patients to CV events. 


\section{References}

1. Montalescot, G. et al. 2013 ESC guidelines on the management of stable coronary artery disease: the task force on the management of stable coronary artery disease of the European Society of Cardiology. Eur Heart J 34, 2949-3003 (2013).

2. Bonaca, M. P. et al. Long-term use of ticagrelor in patients with prior myocardial infarction. $N$ Engl J Med 372, 1791-1800 (2015).

3. Morrow, D. A. et al. Vorapaxar in the secondary prevention of atherothrombotic events. $N$ Engl J Med 366, 1404-1413 (2012).

4. Connolly, S. J. et al. Rivaroxaban with or without aspirin in patients with stable coronary artery disease: an international, randomised, double-blind, placebo-controlled trial. Lancet 391, 205218 (2018).

5. Ridker, P. M. et al. Antiinflammatory therapy with canakinumab for atherosclerotic disease. $N$ Engl J Med 377, 1119-1131 (2017).

6. Cannon, C. P. et al. Ezetimibe added to statin therapy after acute coronary syndromes. $N$ Engl J Med 372, 2387-2397 (2015).

7. Clayton, T. C. et al. Risk score for predicting death, myocardial infarction, and stroke in patients with stable angina, based on a large randomised trial cohort of patients. BMJ 331, 869 (2005).

8. Falk, E., Nakano, M., Bentzon, J. F., Finn, A. V. \& Virmani, R. Update on acute coronary syndromes: the pathologists' view. Eur Heart J 34, 719-728 (2013).

9. Libby, P. \& Theroux, P. Pathophysiology of coronary artery disease. Circulation 111, 34813488 (2005).

10. Arbab-Zadeh, A. \& Fuster, V. The myth of the "vulnerable plaque": transitioning from a focus on individual lesions to atherosclerotic disease burden for coronary artery disease risk assessment. J Am Coll Cardiol 65, 846-855 (2015).

11. Bhatt, D. L. et al. International prevalence, recognition, and treatment of cardiovascular risk factors in outpatients with atherothrombosis. JAMA 295, 180-189 (2006).

12. Kubo, T. et al. Assessment of culprit lesion morphology in acute myocardial infarction: ability of optical coherence tomography compared with intravascular ultrasound and coronary angioscopy. J Am Coll Cardiol 50, 933-939 (2007).

13. $\mathrm{Xu}, \mathrm{Y}$. et al. Prevalence, distribution, predictors, and outcomes of patients with calcified nodules in native coronary arteries: a 3-vessel intravascular ultrasound analysis from Providing Regional Observations to Study Predictors of Events in the Coronary Tree (PROSPECT). Circulation 126, 537-545 (2012).

14. Virmani, R., Kolodgie, F. D., Burke, A. P., Farb, A. \& Schwartz, S. M. Lessons from sudden coronary death: a comprehensive morphological classification scheme for atherosclerotic lesions. Arterioscler Thromb Vasc Biol 20, 1262-1275 (2000).

15. Stone, G. W. et al. A prospective natural-history study of coronary atherosclerosis. N Engl J Med 364, 226-235 (2011).

16. Libby, P., Pasterkamp, G., Crea, F. \& Jang, I. K. Reassessing the mechanisms of acute coronary syndromes: the "vulnerable plaque" and superficial erosion. Circ Res 124, 150-160 (2019).

17. Yeh, R. W. et al. Population trends in the incidence and outcomes of acute myocardial infarction. N. Engl. J. Med 362, 2155-2165 (2010).

18. McManus, D. D. et al. Recent trends in the incidence, treatment, and outcomes of patients with STEMI and NSTEMI. Am J Med 124, 40-47 (2011).

19. Murugiah, K., Nuti, S. V. \& Krumholz, H. M. STEMI care in LMIC: obstacles and opportunities. Glob Heart 9, 429-430 (2014).

20. Murugiah, K. et al. Are non-ST-segment elevation myocardial infarctions missing in China? Eur Heart J Qual Care Clin Outcomes 3, 319-327 (2017).

21. Naghavi, M. et al. From vulnerable plaque to vulnerable patient: a call for new definitions and risk assessment strategies: Part I. Circulation 108, 1664-1672 (2003). 
22. Asakura, M. et al. Extensive development of vulnerable plaques as a pan-coronary process in patients with myocardial infarction: an angioscopic study. J Am Coll Cardiol 37, 1284-1288 (2001).

23. Burke, A. P. et al. Healed plaque ruptures and sudden coronary death: evidence that subclinical rupture has a role in plaque progression. Circulation 103, 934-940 (2001).

24. Kubo, T. et al. The dynamic nature of coronary artery lesion morphology assessed by serial virtual histology intravascular ultrasound tissue characterization. J Am Coll Cardiol 55, 15901597 (2010).

25. Ali, Z. A. et al. Intracoronary optical coherence tomography 2018: current status and future directions. JACC Cardiovasc Interv 10, 2473-2487 (2017).

26. Tarkin, J. M., Joshi, F. R., Rajani, N. K. \& Rudd, J. H. PET imaging of atherosclerosis. Future Cardiol 11, 115-131 (2015).

27. Irkle, A. et al. Identifying active vascular microcalcification by (18)F-sodium fluoride positron emission tomography. Nat Commun 6, 7495 (2015).

28. Buysschaert, I. et al. A variant at chromosome 9 p21 is associated with recurrent myocardial infarction and cardiac death after acute coronary syndrome: the GRACE Genetics Study. Eur Heart J 31, 1132-1141 (2010).

29. Adamson, P. D. et al. High-sensitivity cardiac troponin I and the diagnosis of coronary artery disease in patients with suspected angina pectoris. Circ Cardiovasc Qual Outcomes 11, e004227 (2018).

30. Januzzi, J. L., Jr. et al. High-sensitivity troponin I and coronary computed tomography in symptomatic outpatients with suspected coronary artery disease: insights from the PROMISE trial. JACC Cardiovasc Imaging: doi:10.1016/j.jcmg.2018.01.021 (2018).

31. Lindholm, D. et al. Biomarker-based risk model to predict cardiovascular mortality in patients with stable coronary disease. J Am Coll Cardiol 70, 813-826 (2017).

32. Thomas, M. R. \& Lip, G. Y. H. Novel risk markers and risk assessments for cardiovascular disease. Circ Res 120, 133-149 (2017).

33. Park, K. C., Gaze, D. C., Collinson, P. O. \& Marber, M. S. Cardiac troponins: from myocardial infarction to chronic disease. Cardiovasc Res 113, 1708-1718 (2017).

34. Smith, S. C., Jr. et al. AHA/ACCF secondary prevention and risk reduction therapy for patients with coronary and other atherosclerotic vascular disease: 2011 update: a guideline from the American Heart Association and American College of Cardiology Foundation. Circulation 124, 2458-2473 (2011).

35. Zinman, B. et al. Empagliflozin, cardiovascular outcomes, and mortality in type 2 diabetes. $N$ Engl J Med 373, 2117-2128 (2015).

36. Neal, B. et al. Canagliflozin and cardiovascular and renal events in type 2 diabetes. $N$ Engl $J$ Med 377, 644-657 (2017).

37. Catapano, A. L. et al. 2016 ESC/EAS guidelines for the management of dyslipidaemias. Rev Esp Cardiol (Engl Ed) 70, 115 (2017).

38. Fihn, S. D. et al. 2012 ACCF/AHA/ACP/AATS/PCNA/SCAI/STS guideline for the diagnosis and management of patients with stable ischemic heart disease: a report of the American College of Cardiology Foundation/American Heart Association task force on practice guidelines, and the American College of Physicians, American Association for Thoracic Surgery, Preventive Cardiovascular Nurses Association, Society for Cardiovascular Angiography and Interventions, and Society of Thoracic Surgeons. Circulation 126, e354-e471 (2012).

39. Vaccarino, V. et al. Depression and coronary heart disease: 2018 ESC position paper of the working group of coronary pathophysiology and microcirculation developed under the auspices of the ESC Committee for Practice Guidelines. Eur Heart J: doi:10.1093/eurheartj/ehy913 (2019).

40. Windecker, S. et al. 2014 ESC/EACTS guidelines on myocardial revascularization. Eurolntervention 10, 1024-1094 (2015).

41. Katritsis, D. G., Mark, D. B. \& Gersh, B. J. Revascularization in stable coronary disease: evidence and uncertainties. Nat Rev Cardiol 15, 408-419 (2018).

42. Valgimigli, M. et al. 2017 ESC focused update on dual antiplatelet therapy in coronary artery disease developed in collaboration with EACTS: the Task Force for dual antiplatelet therapy in 
coronary artery disease of the European Society of Cardiology (ESC) and of the European Association for Cardio-Thoracic Surgery (EACTS). Eur Heart J 39, 213-260 (2018).

43. Eikelboom, J. W. et al. Rivaroxaban with or without aspirin in stable cardiovascular disease. $N$ Engl J Med 377, 1319-1330 (2017).

44. Steg, P. G. et al. One-year cardiovascular event rates in outpatients with atherothrombosis. JAMA 297, 1197-1206 (2007).

45. Jernberg, T. et al. Cardiovascular risk in post-myocardial infarction patients: nationwide real world data demonstrate the importance of a long-term perspective. Eur Heart J 36, 1163-1170 (2015).

46. SCOT-HEART Investigators et al. Coronary CT angiography and 5-year risk of myocardial infarction. N Engl J Med 379, 924-933 (2018).

47. Rapsomaniki, E. et al. Prognostic models for stable coronary artery disease based on electronic health record cohort of 102023 patients. Eur Heart J 35, 844-852 (2014).

48. Rapsomaniki, E. et al. Using big data from health records from four countries to evaluate chronic disease outcomes: a study in 114364 survivors of myocardial infarction. Eur Heart $J$ Qual Care Clin Outcomes 2, 172-183 (2016).

49. Sachdev, M. et al. The prognostic importance of comorbidity for mortality in patients with stable coronary artery disease. J Am Coll Cardiol 43, 576-582 (2004).

50. Eisen, A. et al. Angina and future cardiovascular events in stable patients with coronary artery disease: insights from the Reduction of Atherothrombosis for Continued Health (REACH) Registry. J Am Heart Assoc 5, e004080 (2016).

51. Yeo, K. K. et al. Comparative analysis of recurrent events after presentation with an index myocardial infarction or ischemic stroke. Eur Heart J Qual Care Clin Outcomes 3, 234-242 (2016).

52. Fox, K. A. A. et al. Time course of events in acute coronary syndromes: implications for clinical practice from the GRACE registry. Nat Clin Pract Cardiovasc Med 5, 580-589 (2008).

53. Ringleb, P. A. et al. Benefit of clopidogrel over aspirin is amplified in patients with a history of ischemic events. Stroke 35, 528-532 (2004).

54. Bhatt, D. L. et al. Comparative determinants of 4-year cardiovascular event rates in stable outpatients at risk of or with atherothrombosis. JAMA 304, 1350-1357 (2010).

55. O'Donoghue, M. L. et al. Effect of darapladib on major coronary events after an acute coronary syndrome: the SOLID-TIMI 52 randomized clinical trial. JAMA 312, 1006-1015 (2014).

56. Marso, S. P. et al. Liraglutide and cardiovascular outcomes in type 2 diabetes. N Engl J Med 375, 311-322 (2016).

57. Holman, R. R. et al. Effects of once-weekly exenatide on cardiovascular outcomes in type 2 diabetes. N Engl J Med 377, 1228-1239 (2017).

58. Suarez, C. et al. Influence of polyvascular disease on cardiovascular event rates. Insights from the REACH Registry. Vasc Med 15, 259-265 (2010).

59. Bansilal, S. et al. Ticagrelor for secondary prevention of atherothrombotic events in patients with multivessel coronary disease. J Am Coll Cardiol 71, 489-496 (2018).

60. Wilson, P. W. et al. An international model to predict recurrent cardiovascular disease. Am. J. Med 125, 695-703 (2012).

61. CAPRIE Steering Committee A randomised, blinded, trial of clopidogrel versus aspirin in patients at risk of ischaemic events (CAPRIE). Lancet 348, 1329-1339 (1996).

62. Bhatt, D. L. et al. Superiority of clopidogrel versus aspirin in patients with prior cardiac surgery. Circulation 103, 363-368 (2001).

63. Kosova, E. C. et al. Vorapaxar in patients with coronary artery bypass grafting: Findings from the TRA $2^{\circ} \mathrm{P}$-TIMI 50 trial. Eur Heart J Acute Cardiovasc Care 6, 164-172 (2017).

64. Yap, C. H. et al. Contemporary results show repeat coronary artery bypass grafting remains a risk factor for operative mortality. Ann Thorac Surg 87, 1386-1391 (2009).

65. Cavender, M. A. et al. Impact of diabetes mellitus on hospitalization for heart failure, cardiovascular events, and death: outcomes at 4 years from the REduction of Atherothrombosis for Continued Health (REACH) Registry. Circulation 132, 923-931 (2015).

66. Virmani, R., Burke, A. P. \& Kolodgie, F. Morphological characteristics of coronary atherosclerosis in diabetes mellitus. Can J Cardiol 22 Suppl B, 81B-84B (2006). 
67. Bhatt, D. L. et al. Amplified benefit of clopidogrel versus aspirin in patients with diabetes mellitus. Am J Cardiol 90, 625-628 (2002).

68. Cavender, M. A. et al. Vorapaxar in patients with diabetes mellitus and previous myocardial infarction: findings from the Thrombin Receptor Antagonist in Secondary Prevention of Atherothrombotic Ischemic Events-TIMI 50 trial. Circulation 131, 1047-1053 (2015).

69. Brooks, M. M. et al. Clinical and angiographic risk stratification and differential impact on treatment outcomes in the Bypass Angioplasty Revascularization Investigation 2 Diabetes (BARI 2D) trial. Circulation 126, 2115-2124 (2012).

70. Dumaine, R. L. et al. Renal function, atherothrombosis extent, and outcomes in high-risk patients. Am Heart J 158, 141-148 e141 (2009).

71. Kalra, P. R. et al. Impact of chronic kidney disease on use of evidence-based therapy in stable coronary artery disease: a prospective analysis of 22,272 patients. PLoS One 9, e102335 (2014).

72. Hill, N. R. et al. Global prevalence of chronic kidney disease - a systematic review and metaanalysis. PLoS One 11, e0158765 (2016).

73. Cai, Q., Mukku, V. K. \& Ahmad, M. Coronary artery disease in patients with chronic kidney disease: a clinical update. Curr Cardiol Rev 9, 331-339 (2013).

74. Briasoulis, A. \& Bakris, G. L. Chronic kidney disease as a coronary artery disease risk equivalent. Current cardiology reports 15, 340 (2013).

75. Bernaudo, D. et al. Renal function and short-term outcome in stable outpatients with coronary, cerebrovascular or peripheral artery disease. Atherosclerosis 229, 258-262 (2013).

76. Magnani, G. et al. Efficacy and safety of ticagrelor for long-term secondary prevention of atherothrombotic events in relation to renal function: insights from the PEGASUS-TIMI 54 trial. Eur Heart J 37, 400-408 (2016).

77. Gheorghiade, M. et al. Navigating the crossroads of coronary artery disease and heart failure. Circulation 114, 1202-1213 (2006).

78. GBD 2016 Disease and Injury Incidence and Prevalence Collaborators Global, regional, and national incidence, prevalence, and years lived with disability for 328 diseases and injuries for 195 countries, 1990-2016: a systematic analysis for the Global Burden of Disease Study 2016. Lancet 390, 1211-1259 (2017).

79. Gheorghiade, M. et al. Systolic blood pressure at admission, clinical characteristics, and outcomes in patients hospitalized with acute heart failure. JAMA 296, 2217-2226 (2006).

80. Seferovic, P. M. et al. Type 2 diabetes mellitus and heart failure: a position statement from the Heart Failure Association of the European Society of Cardiology. Eur J Heart Fail 20, 853-872 (2018).

81. Jensen, L. O. et al. Influence of diabetes mellitus on clinical outcomes following primary percutaneous coronary intervention in patients with ST-segment elevation myocardial infarction. Am J Cardiol 109, 629-635 (2012).

82. Ritsinger, V., Saleh, N., Lagerqvist, B. \& Norhammar, A. High event rate after a first percutaneous coronary intervention in patients with diabetes mellitus: results from the Swedish coronary angiography and angioplasty registry. Circ Cardiovasc Interv 8, e002328 (2015).

83. Weitz, J. I. Insights into the role of thrombin in the pathogenesis of recurrent ischaemia after acute coronary syndrome. Thromb Haemost 112, 924-931 (2014).

84. Casa, L. D., Deaton, D. H. \& Ku, D. N. Role of high shear rate in thrombosis. J Vasc Surg 61, 1068-1080 (2015).

85. Bhatt, D. L. et al. Clopidogrel and aspirin versus aspirin alone for the prevention of atherothrombotic events. N Engl J Med 354, 1706-1717 (2006).

86. Bhatt, D. L. et al. Patients with prior myocardial infarction, stroke, or symptomatic peripheral arterial disease in the CHARISMA trial. J Am Coll Cardiol 49, 1982-1988 (2007).

87. Hirsh, J. \& Bhatt, D. L. Comparative benefits of clopidogrel and aspirin in high-risk patient populations: lessons from the CAPRIE and CURE studies. Arch Intern Med 164, 2106-2110 (2004).

88. Bhatt, D. L. et al. Reduction in ischemic events with ticagrelor in diabetic patients with prior myocardial infarction in PEGASUS-TIMI 54. J Am Coll Cardiol 67, 2732-2740 (2016). 
89. Magnani, G. et al. Efficacy and safety of edoxaban compared with warfarin in patients with atrial fibrillation and heart failure: insights from ENGAGE AF-TIMI 48. Eur J Heart Fail 18, 1153-1161 (2016).

90. Rothberg, M. B., Celestin, C., Fiore, L. D., Lawler, E. \& Cook, J. R. Warfarin plus aspirin after myocardial infarction or the acute coronary syndrome: meta-analysis with estimates of risk and benefit. Ann. Intern. Med 143, 241-250 (2005).

91. Testa, L. et al. Adjusted indirect meta-analysis of aspirin plus warfarin at international normalized ratios 2 to 3 versus aspirin plus clopidogrel after acute coronary syndromes. Am. J. Cardiol 99, 1637-1642 (2007).

92. Alexander, J. H. et al. Apixaban with antiplatelet therapy after acute coronary syndrome. $\mathrm{N}$ Engl J Med 365, 699-708 (2011).

93. Mega, J. L. et al. Rivaroxaban in patients with a recent acute coronary syndrome. N Engl J Med 366, 9-19 (2012).

94. Ohman, E. M. et al. Clinically significant bleeding with low-dose rivaroxaban versus aspirin, in addition to P2Y12 inhibition, in acute coronary syndromes (GEMINI-ACS-1): a double-blind, multicentre, randomised trial. Lancet 389, 1799-1808 (2017).

95. Stachon, P., Ahrens, I., Bode, C. \& Zirlik, A. Dual pathway therapy in acute coronary syndrome. $J$ Thromb Thrombolysis 42, 254-260 (2016).

96. Bayer AG. Xarelto ${ }^{\circledR}$ (rivaroxaban) Summary of Product Characteristics. http://www.ema.europa.eu/docs/en GB/document library/EPAR Product Information/human/000944/WC500057108.pdf (2018).

97. Janssen Pharmaceuticals Inc. Xarelto ${ }^{\circledR}$ (rivaroxaban) Prescribing Information - SUPERSEDED. http://www.janssenlabels.com/package-insert/product-monograph/prescribinginformation/XARELTO-pi.pdf (2019).

98. Dewilde, W. J. et al. Use of clopidogrel with or without aspirin in patients taking oral anticoagulant therapy and undergoing percutaneous coronary intervention: an open-label, randomised, controlled trial. Lancet 381, 1107-1115 (2013).

99. Anand, S. S. et al. Rivaroxaban with or without aspirin in patients with stable peripheral or carotid artery disease: an international, randomised, double-blind, placebo-controlled trial. Lancet 391, 219-229 (2018).

100. Darmon, A. et al. External applicability of the COMPASS trial: an analysis of the reduction of atherothrombosis for continued health (REACH) registry. Eur Heart J 39, 750-757a (2018).

101. Bai, J., Gong, L. L., Li, Q. F. \& Wang, Z. H. Long-term efficacy and safety of proprotein convertase subtilisin/kexin 9 monoclonal antibodies: A meta-analysis of 11 randomized controlled trials. J Clin Lipidol 12, 277-291 e273 (2018).

102. Silverman, M. G. et al. Association between lowering LDL-C and cardiovascular risk reduction among different therapeutic interventions: a systematic review and meta-analysis. JAMA 316, 1289-1297 (2016).

103. Schiele, F. et al. A consensus statement on lipid management after acute coronary syndrome. Eur Heart J Acute Cardiovasc Care 7, 532-543 (2018).

104. Ridker, P. M. et al. Low-dose methotrexate for the prevention of atherosclerotic events. New England Journal of Medicine DOI: 10.1056/NEJMoa1809798, (2019).

105. Kaul, S. Mitigating cardiovascular risk in type 2 diabetes with antidiabetes drugs: a review of principal cardiovascular outcome results of EMPA-REG OUTCOME, LEADER, and SUSTAIN-6 trials. Diabetes Care 40, 821-831 (2017).

106. Marso, S. P. et al. Liraglutide and cardiovascular outcomes in type 2 diabetes. N Engl J Med 375, 311-322 (2016).

107. Marso, S. P. et al. Semaglutide and cardiovascular outcomes in patients with type 2 diabetes. $N$ Engl J Med 375, 1834-1844 (2016).

108. Steg, P. G. et al. Prevalence of anginal symptoms and myocardial ischemia and their effect on clinical outcomes in outpatients with stable coronary artery disease: data from the International Observational CLARIFY Registry. JAMA Intern Med 174, 1651-1659 (2014).

109. Steg, P. G. et al. Women and men with stable coronary artery disease have similar clinical outcomes: insights from the international prospective CLARIFY registry. Eur Heart J 33, 28312840 (2012). 
110. Fox, K. A. A. et al. Should patients with acute coronary disease be stratified for management according to their risk? Derivation, external validation and outcomes using the updated GRACE risk score. BMJ Open 4, e004425 (2014).

111. Saar, A. et al. The risk-treatment paradox in non-ST-elevation myocardial infarction patients according to their estimated GRACE risk. Int J Cardiol doi: 10.1016/j.ijcard.2018.08.015, (2018).

112. Bohula, E. A. et al. Atherothrombotic risk stratification and ezetimibe for secondary prevention. J Am Coll Cardiol 69, 911-921 (2017).

113. Bobadilla, R. V. Acute coronary syndrome: focus on antiplatelet therapy. Crit Care Nurse 36, 15-27 (2016).

114. Fox, K. A. A. et al. Anti-thrombotic options for secondary prevention in patients with chronic atherosclerotic vascular disease: what does COMPASS add? Eur Heart $J$ doi:

10.1093/eurheartj/ehy347, (2018). 


\section{Acknowledgements}

The authors would like to acknowledge Hayley Dawson PhD, who provided editorial support with funding from Bayer AG and Janssen Scientific Affairs, LLC.

\section{Competing interests}

Editorial support was funded by Bayer AG and Janssen Scientific Affairs, LLC.

K.F. has received speaker honoraria and consulting fees from AstraZeneca, Bayer/Janssen, Sanofi/Regeneron, and Verseon. M.M. has received speaker honoraria and consulting fees for his participation in executive committees, advisory boards, or speeches from Amgen, Bayer, Fresenius, Novartis, and Servier. J.M. has received speaker honoraria and consulting fees from Amgen, AstraZeneca, Bayer, Boehringer Ingelheim, Merck Sharp \& Dohme, Novartis, and Servier. D.A. has received speaker honoraria and consulting fees from Amgen, AstraZeneca, Bayer, Boehringer Ingelheim, Bristol-Myers Squibb/Pfizer, Merck Sharp \& Dohme, and Novartis. 


\section{Tables}

Table 1. Residual risk of MACE in patients with chronic CAD (data from registries)

\begin{tabular}{lll}
\hline $\begin{array}{l}\text { Registry (patient population or } \\
\text { subgroup) }\end{array}$ & $\begin{array}{l}\% \text { of the registry } \\
\text { population }\end{array}$ & Residual risk of MACE \\
\hline
\end{tabular}

\section{REACH registry}

Patients with $C A D^{11}$

$59.3 \%$

$4.5 \%$

Patients with CAD and 'stable'

$52 \%$

angina $^{50}$

48.4

Patients with CAD and a previous

CV event ${ }^{54}$

$24.8 \%$

Patients with CAD and concomitant

disease in other vascular beds ${ }^{11,44}$

Patients with CAD and T2DM65

$43.6 \%$

4-year incidence: $16.3 \%$ in patients with 'stable' angina versus $14.2 \%$ in patients without

4-year incidence: $18.3 \%$ in patients with a previous ischaemic event versus $12.2 \%$ without

1-year incidence: $~ 7.0 \%$ versus $4.5 \%$ in the overall CAD population

4-year incidence: $16.5 \%$ in patients with T2DM versus $13.1 \%$ without

Patients at risk of atherothrombotic

$34.7 \%$ had moderate-

1-year incidence: $\mathrm{CV}$ death ranged from $1.7 \%$ in patients disease and $C K D^{70}$ to-severe CKD with $\mathrm{CrCl} \geq 90 \mathrm{~mL} / \mathrm{min}$ to $3.8 \%$ in patients with a $\mathrm{CrCl}<30$ $\mathrm{mL} / \mathrm{min}$ ( $\mathrm{p}$-trend $<0.01$ ); the risk of non-fatal $\mathrm{MI}$ ranged from $1.0 \%$ to $2.0 \%$ ( $p$-trend $<0.01$ ), and the risk of stroke from $1.5 \%$ to $2.0 \%$ ( $p$-trend 0.1 )

$\mathrm{CAD}$ and $\mathrm{HF}^{54}$

$13.6 \%$

$71 \%$ increased risk of MACE (4-year data)

\section{CLARIFY registry}

Patients with CAD and 'stable' angina ${ }^{108}$

Patients with $C A D$ and a previous

CV event ${ }^{109}$

Patients with $C A D$ and $C K D^{71}$
$20.0 \%$

$59.7 \%$ of patients enrolled had a previous MI

$22.1 \%$ of patients had NR an eGFR

$<60 \mathrm{~mL} / \mathrm{min} / 1.73 \mathrm{~m}^{2}$

NR 2-year incidence: $4.2 \%$ in patients with 'stable' angina versus $2.7 \%$ in patients without

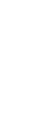

\section{FRENA registry}


$27.3 \%$ of patients had an eGFR

$<60 \mathrm{~mL} / \mathrm{min} / 1.73 \mathrm{~m}^{2}$
Risk of $\mathrm{Ml}: 1.38 \%$ per year in patients with eGFR $>60$ $\mathrm{mL} / \mathrm{min} / 1.73 \mathrm{~m}^{2}$ versus $5.79 \%$ per year in patients with eGFR $30-60 \mathrm{~mL} / \mathrm{min} / 1.73 \mathrm{~m}^{2}$ versus $18.8 \%$ per year in patients with eGFR $<30 \mathrm{~mL} / \mathrm{min} / 1.73 \mathrm{~m}^{2}$

CAD, coronary artery disease; CKD, chronic kidney disease; $\mathrm{CrCl}$, creatinine clearance; $\mathrm{CV}$, cardiovascular; eGFR, estimated glomerular filtration rate; HF, heart failure; MACE, major adverse cardiovascular events; MI, myocardial infarction; NR, not reported; T2DM, type 2 diabetes mellitus. 
Table 2. Risk scores for predicting events in patients with atherothrombosis

\begin{tabular}{|c|c|c|c|}
\hline & Risk factors & Outcome & Interpretation \\
\hline REACH score ${ }^{\star 60}$ & $\begin{array}{ll}\text { - } & \text { Age } \\
\text { - } & \mathrm{AF} \\
\text { - } & \mathrm{ASA} \text { therapy } \\
\text { - } & \mathrm{BMI} \\
\text { - } & \mathrm{CHF} \\
\text { - } & \text { Country of residence } \\
\text { - } & \mathrm{CV} \text { event }<1 \text { year } \\
\text { - } & \text { Diabetes } \\
\text { mellitusNumber of } \\
\text { vascular beds } \\
\text { - } \text { affected } \\
\text { - Sex } \\
\text { - Statin therapy }\end{array}$ & $\begin{array}{l}\text { Risk of CV death } \\
\text { and recurrent } \mathrm{CV} \\
\text { events at } 20 \\
\text { months }\end{array}$ & $\begin{array}{l}\text { Example } \\
\text { Scores 0-8: }<1 \% \\
\text { Score 10: } 1.4 \% \\
\text { Score } \geq 26:>50 \%\end{array}$ \\
\hline Modified REACH score ${ }^{\star 60,100}$ & $\begin{array}{l}\text { - } \mathrm{AF} \\
\text { - } \mathrm{Age} \\
\text { - } \mathrm{ASA} \text { therapy } \\
\text { - } \mathrm{BMI} \\
\text { - } \mathrm{CHF} \\
\text { - } \text { Country of residence } \\
\text { - } \mathrm{CV} \text { event }<1 \text { year } \\
\text { - } \text { Diabetes mellitus } \\
\text { - } \text { Number of vascular } \\
\text { - } \text { beds affected } \\
\text { - Sex } \\
\text { - Smoking status } \\
\text { Statin therapy }\end{array}$ & $\begin{array}{l}\text { Recurrent CV } \\
\text { events }\end{array}$ & $\begin{array}{l}\text { Scores range from } 0 \\
\text { to }>29 \text { (low to high } \\
\text { risk) }\end{array}$ \\
\hline $\begin{array}{l}\text { Updated GRACE score }{ }^{\ddagger 110} \\
\text { (https://www.mdcalc.com/grace- } \\
\text { acs-risk-mortality-calculator) }\end{array}$ & $\begin{array}{ll}\text { - } & \text { Age } \\
\text { - } & \text { Creatinine } \\
\text { - } & \text { Systolic blood } \\
& \text { pressure } \\
\text { - } & \text { Pulse } \\
\text { - } & \text { Cardiac arrest at } \\
& \text { admission (Yes/No) }\end{array}$ & $\begin{array}{l}\text { Risk of death or } \\
\text { death/MI following } \\
\text { an initial ACS }\end{array}$ & $\begin{array}{l}\text { Example (following } \\
\text { NSTEMI) } \\
\text { Low }(<4 \%) \\
\text { Intermediate (4- } \\
12 \%) \\
\text { High (>12\%) }\end{array}$ \\
\hline
\end{tabular}


- ST segmented deviation on EKG? (Yes/No)

- Abnormal cardiac enzymes? (Yes/No)

- Killip class (No CHF, rales and/or JVD, pulmonary oedema, cardiogenic shock)

TRA $2^{\circ} \mathrm{P}$ score $\$ 112$

- Age $\geq 75$ years old

Risk of MACE at 7 Low: $0-1$

- $\mathrm{CHF}$ years

Intermediate: 2

- Diabetes mellitus

- eGFR <60 High: $\geq 3$ (maximum score $=9$ )

- Hypertension

- PAD

- Prior stroke

- Prior CABG

- Smoking

${ }^{*}$ The number of points allocated per factor is categorical and full details are provided in Wilson et al, 2012 (e.g. there are 14 age categories allocated points ranging from 0 to 14 ) ${ }^{60}$; *the updated GRACE score generates absolute percentage risks ${ }^{110}$ and the calculator is available online at: https://www.mdcalc.com/grace-acs-risk-mortality-calculator; ${ }^{\S}$ all risk factors are allocated 1 point ${ }^{112}$.

ACS, acute coronary syndrome; AF, atrial fibrillation; ASA, acetylsalicylic acid; BMI, body mass index; CABG, coronary artery bypass grafting; $\mathrm{CHF}$, congestive heart failure; $\mathrm{CV}$, cardiovascular; eGFR, estimated glomerular filtration rate; EKG, electrocardiogram; JVD, jugular venous distention; MACE, major adverse cardiovascular events; MI, myocardial infarction; NSTEMI, non-ST-elevation myocardial infarction; PAD, peripheral artery disease. 


\section{Figure legends}

Figure 1. Expected and observed probabilities of dying or sustaining myocardial infarction or disabling stroke in 5 years by tenth of risk score for patients with 'stable' angina?.

Figure 2. A) The variable clinical manifestations of $C A D^{113}$. B) The risk of thrombosis at the site of plaque rupture ${ }^{10}$.

ACS, acute coronary syndrome; CAD, coronary artery disease.

Figure 3. Event prevention in patients with chronic $C A D^{1,34}$.

ACE, angiotensin-converting enzyme; ARB, angiotensin receptor blocker; ASA, acetylsalicylic acid; CAD, coronary artery disease.

Figure 4. The persistent risk of MACE after a CV event as demonstrated by observational ${ }^{45,48,54}$ and $\mathrm{RCT}^{55-57}$ data.

*Defined as non-fatal stroke, non-fatal MI, or CV death; "defined as CHD death, MI, or urgent coronary revascularization for $\mathrm{Ml}$.

ACS, acute coronary syndrome; CHD, congestive heart disease; CV, cardiovascular; MACE, major adverse cardiovascular events; MI, myocardial infarction; RCT, randomized controlled trial.

Figure 5. Residual risk of MACE and bleeding events in patients with chronic CAD treated with various antithrombotic regimens (data from $\mathrm{RCTs}$ ) 2,3,43,61,85. ASA, acetylsalicylic acid; bid, twice daily; CAD, coronary artery disease; CV, cardiovascular; GI, gastrointestinal; ICH, intracranial haemorrhage; MACE, major adverse cardiovascular events; MI, myocardial infarction; PAD, peripheral artery disease; RCT, randomized controlled trial.

Figure 6. The impact of different regimens in the context of proven secondary prevention regimens (ASA, lipid lowering, blood pressure, and ACE inhibitors) ${ }^{2,3,43,61,85,114}$. Patient eligibility was as follows. CAPRIE: patients with atherosclerotic vascular disease manifested as either recent ischaemic stroke, recent MI, or symptomatic PAD; CHARISMA: patients $\geq 45$ years of age with multiple atherothrombotic risk factors and/or, documented CAD, cerebrovascular disease or symptomatic PAD; PEGASUS: patients with spontaneous MI 1-3 years before enrolment, $\geq 50$ years old plus one additional risk factor (age $\geq 65$ years, diabetes mellitus requiring medication, a second prior spontaneous $\mathrm{MI}$, multivessel CAD, or 
$\mathrm{eCrCl}<60 \mathrm{~mL} / \mathrm{min}$ ); TRA $2^{\circ} \mathrm{P}-\mathrm{TIMI} 50$ : patients with a history of atherosclerosis, defined as a spontaneous $\mathrm{MI}$ or ischaemic stroke within 2 weeks to 12 months or PAD associated with a history of intermittent claudication in conjunction with either an $\mathrm{ABI}<0.85$ or previous revascularization for limb ischaemia; COMPASS: patients who met the criteria for CAD and/or PAD (for patients with CAD and $<65$ years old were also required to have documentation of atherosclerosis involving $\geq 2$ vascular beds or to have at least two additional risk factors).

$\mathrm{ABI}$, ankle brachial index; $\mathrm{ACE}$, angiotensin-converting enzyme; ASA, acetylsalicylic acid; ATT, Antithrombotic Treatment Trialists'; CAD, coronary artery disease; $\mathrm{eCrCl}$, estimated creatinine clearance; MACE, major adverse cardiovascular events; MI, myocardial infarction; PAD, peripheral artery disease. 